\title{
TRENDS AND ISSUES IN COMMUNITY TELECARE IN THE UNITED KINGDOM
}

\author{
R. Sethi*, D. Azzi*, R. Khusainov* \\ *Dept. of Electronic and Computer Eng., University of Portsmouth, UK. Correspondence email: richie.sethi@ port.ac.uk
}

Keywords: telecare, population, trends, Government, prediction

\begin{abstract}
With an increasing elderly population putting a strain on the cost of care, and the social, health and cost benefits of using community social care and telecare services, telecare is experiencing growth. This paper discusses trends in demand for telecare and issues with service provision and mainstreaming services and evaluates access, service reliability, the market, standards, interoperability and technology developments.
\end{abstract}

\section{Introduction}

Community telecare for the elderly user is focussed on the provision of chronic or acute social care or nursing, including social alarms, fall monitoring, dementia care, and longer term wellbeing management. The health issues associated with ageing are well understood in terms of mental health and physical disorders [1]. Elderly care has specific and unique challenges and residential and community care models are well defined [1]. Various social factors come into play, including attitudes towards the elderly, social pressures and the growing population [2]. There has been a move away from the state (NHS) providing care since 1980s, towards the private and not for profit sectors [1]. Telecare makes care provision more efficient and cost effective and may assist to reduce, delay and shorten the duration of hospital admissions, improve quality of life and patient safety and promote independence, which has consequently lead to the popularisation of the technology in recent years, supported by Government initiatives and decreasing technology cost.

The introduction of next-generation IP-based technology is hindered by the lack of a national telecare infrastructure, issues with standards and interoperability, and reluctance to move away from analogue technology. However, with lowering costs and improvements in technical standards, the technology is expected to grow significantly.

With many activities taking place in technology development and deployment, standardisation and interoperability, formation of care strategies and allocation of funds, it is important to understand both the current situation and trends in telecare, to direct future efforts. This paper hence carries out a review of published work, government reports and other data to identify the various market, research, technological, standardisation and social trends in community telecare in the United Kingdom and examine issues.

The contributions of this paper are as follows: A discussion of the trends in population and service demand, and issues with the provision and mainstreaming of services; an evaluation of access, service reliability, the market and standards, interoperability and technology development; and finally, a prediction of the future of telecare for the next decade.

\section{Telecare and Social Care}

\subsection{Background}

Telecare is often cited as an extension of social care services [3]. The Department of Health states that 'Telecare is as much about the philosophy of dignity and independence as it is about equipment and services' [4]. It follows that telecare is also influenced by inequalities within social care provision and associated challenges [3].

There have been notable improvements in care provision to the elderly. These include improvements in drug therapies, equipment and health care practice (resulting in a sustained increase in life expectancy and higher rate of survival from illness or injury), the establishment of 'rights and responsibilities in relation to social care, placing statutory obligations on local authorities to provide care services', the transfer of those with mental health problems from dedicated institutions to community supported-living schemes and finally, free access to a national health service for clinical care [5].

Still, there are significant challenges in social care, arising out of pressures on social care provision (and hence telecare) due to a growing elderly population and strained economic resources [6].

Telecare has been shown to have a significant positive impact on social care provision in terms of helping people live independently in the community for longer, supporting unpaid carers by assisting their care provision, better physical health from reduced numbers of falls, accidents and admissionrelated secondary infections and significant financial saving due to improved response-time, elimination of redundant practices, reduction in hospital admissions and savings as a result of the relatively lower cost of telecare compared to direct care [5]. Williams [7] reports that "no study [of telecare] has yet demonstrated any negative or adverse effects' and Yeandle [5] further argues that 'telecare can 
sustain people with a wide range of conditions in their own homes, and offer support to their carers'.

\subsection{Demand and Supply}

The Office of National Statistics [2] estimates that the UK population will reach $63 \mathrm{~m}$ by 2012 , rising to between 66 and $72.5 \mathrm{~m}$ by 2025 , with the proportion of the population over 65 rising from $19.3 \%$ in $2012(12.2 \mathrm{~m})$, to between $19.5 \%$ and $21.5 \%$ by 2025 (12.9m to $15.6 \mathrm{~m}$ ) (see figure 1 ). The population therefore will become older, with the median age rising from 40 to 41 by 2025 [2].

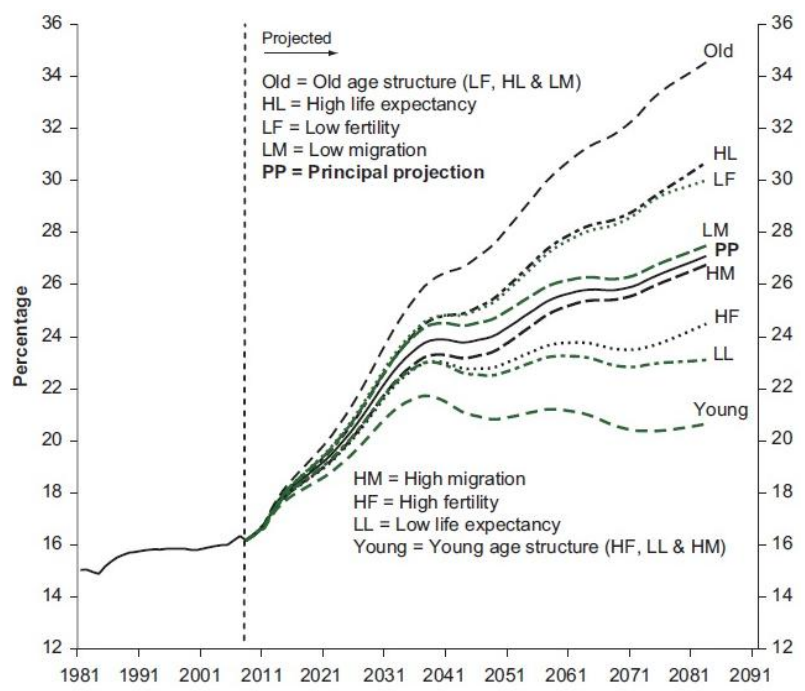

Figure 1: Proportion of UK Population Aged Over 65 [2]

It is estimated that in the $65-84$ age group, $1.16 \mathrm{~m}$ people live alone and with a limiting long-term illness, which is set to rise by $38 \%$ to $1.6 \mathrm{~m}$ in 2025 . Those above the age of 85 are more likely to live alone and have poorer health. In this age group, $37 \%$ of men and $55 \%$ of women live alone [8]. Of these, most have a limiting long-term illness (70\% and $78 \%$ for men and women respectively) and a third are 'persistently in poor general health' (32\% and 36\% respectively) [9]. It is estimated that the number of people aged 85 or above will increase by $70 \%$ to $1.9 \mathrm{~m}$ in 2025 and that those who live alone and have a long-term limiting illness will rise by the same proportion to 'almost 686,000 in 2025 ' [5].

As telecare is most beneficial to an older age group which live alone (usually with a smaller informal support network) and have a limiting long-term illness or disability including mental-health problems, the predicted rise in the size of this group therefore will therefore have a significant impact on telecare provision, or lack thereof in the future.

Parallels can be drawn with community care provision and support and estimates for this provision in the future. Most care support is provided informally, with $4.85 \mathrm{~m}$ unpaid carers providing support to friends and family in England in 2001, with the numbers of new carers higher than the proportion of those who cease their caring role as a result of deaths or changes in the caring situation [5]. Of the $4.85 \mathrm{~m}, 1.6 \mathrm{~m}$ were reported to be of working age and caring for 20 or more hours a week [5] and it has been demonstrated that this care provision 'is associated with a deterioration in their health and financial situation' [5]. Furthermore, many give up paid work or reduce hours of employment, reducing the general economic output of the working population. There is evidence which suggests that telecare can assist unpaid carers in regular employment, by freeing up their time and delegating certain responsibilities, such as monitoring, to automated systems. Older persons unable to do various tasks and receiving support from unpaid carers are significantly less dependent on social services or paid help- 33\% less for bathing/showering, $17 \%$ for domestic tasks and 23\% for practical activities [6], figures which can be further enhanced by the use of telecare [3].

With only about $6 \%$ of those above the age of 65 in receipt of 'a council-supported homecare service' [5], a significant proportion of the elderly population is not eligible for, or are for other reasons not receiving state-supported homecare services, which includes telecare services. It is estimated that the average pensioner, receiving 10 hours of domiciliary care services per week 'will exhaust 50\% of their annual income' on care services alone and that the cost overhead of telecare may not be acceptable to some, particularly state pensioners, who receive an annual income which is less than half of that of the average pensioner, although telecare may be subsidised to some groups [3]. It is difficult to estimate the number of people currently receiving telecare services at this time due to a proliferation of private equipment and service providers. Based on figures that are available, it is estimated that up to $1.7 \mathrm{~m}$ people in the UK as a whole have access to a telecare service (2010 data) [10], of which fewer than $1 \%$ use "nextgeneration' equipment.

\subsection{Mainstreaming Telecare}

Although 'Mainstreaming telecare would massively increase its impact' [5], progress is held back because of a lack of awareness of the benefits of telecare amongst those responsible for providing services, strict eligibility criteria [5], lack of consistency in service provision between areas and lastly, shortages in funding [3]. Yeandle [5] reports that at the current rate of growth, telecare will never be available to all those above the age of 85 and argues that since telecare offers a critical contribution to the health and social care system, it must be adopted on a larger scale at a faster pace, to meet growing demand.

Recent Government initiatives have recognised the health and economic benefits of telecare, for example the new Scottish care policy framework which 'shifts the balance of care' towards community and assistive technology, provided $£ 16.35 \mathrm{~m}$ to support telecare growth in Scotland between 2006 and 2008 [10], which benefited over 6,700 of those aged over 65 and which lead to efficiency savings of over $£ 11 \mathrm{~m}$ [10]. $£ 80 \mathrm{~m}$ was allocated to local councils in England in the form of preventative technology grants (for telecare innovations) during the same two year period [11], highlighting the high level of state-investment in telecare in the UK. 
Furthermore, funded pilot schemes running across the UK aim to develop national infrastructure and encourage innovation in telecare services [10]. The Department of Health's Whole System Demonstrator (WSD) is an ongoing field trial which began in 2007, the aim of which is to develop an evidence-base for telehealth and telecare and which has resulted in growth in local telecare service provision [11].

\section{Technology}

The most common form of telecare is the community alarm [10], which consists of an emergency pendant. Emergency calls are sent via a gateway to a remote call centre, which makes contact with the person in need and makes an intervention decision. Such 'first-generation' equipment does not contain a prolific amount of sensors for data-intensive applications and uses analogue telephone lines for communication. 'Next-generation' equipment uses IP-based digital technology to send information (such as medical and event information) to a remote server for processing and may use data-mining techniques, to identify behaviour patterns and anomalies in these, to predict and therefore pre-empt possible situations requiring care-intervention.

\subsection{Access}

Advances in medical technology have made telecare a costfeasible technology. A very real problem with providing telecare in the modern age, however, is that the infrastructure for its use may be unavailable in some areas [3]. Two practical considerations are that not everyone has access to a telephone which they can readily use and the quality of these lines is uncertain; if the patient and the telecare team cannot communicate, there is little value in subscribing to telecare services [3]. The UK also lags behind some EU countries and the likes of USA, Korea and Japan in high-speed Internet penetration and availability [12], in spite of having prices lower than the EU average [13]. This has contributed to lack of progress of next-generation telecare which involves 'the prediction of possible acute situations' [14] from sensory data and which involves large amounts of data. Currently, only $4 \%$ of over-65s have access to the Internet in any form in their homes [15]. This means that providers may have to add extra costs (including Internet fees) for anything other than the most basic telecare [3]. There are however, technical issues with the fitness of purpose of IP networks for safety-critical services such as social alarms. The Telecare Services Association (TSA), in partnership with the Continua Health Alliance is working to develop standards for telecare over IP, including the establishment of a 'certifiable technical solution' [16].

\subsection{Service Reliability}

The uptake of IP-based telecare services in the UK will be contingent upon guarantees of reliability of the telecommunications link [3]. Although a private circuit offers better reliability than consumer broadband Internet, the former is cost-prohibitive and it follows that ISPs which implement Quality of Service techniques to reserve bandwidth for telecare services will stand to gain, as the industry moves away from analogue telephony towards digital services [3].

\subsection{Market Forces}

There are differences between the UK and the USA in terms of the market share of the commercial enterprises involved and nature of demand. Whilst large multinational organisations operate in both markets, the telecare (in social care) sector in the UK, is largely dominated by SMEs, and demand tends to be driven by public and private purchasing consortia (health trusts, housing and care providers). Meanwhile, in the comparative sector in the USA, multinational organisations have a greater market presence, and direct consumer marketing is more prevalent. The differences may, in part, be attributed to the differences in healthcare provision (state vs. private). Continental Europe and the UK have experienced an increase in popular consumer telecare products and services, with IP-based services more prevalent in the former due, in part, to higher broadband penetration and availability (as discussed above).

\subsection{Standards, Interoperability and Technology Development}

Finally, there is a problem with a lack of standards and interoperability and thus development of next-generation IPbased technology. Most commercial remote monitoring protocols are proprietary [17]. This, along with a lack of specific standards for telecare, has resulted in interoperability issues. Telecare devices are rarely compatible between manufacturers and do not work outside of users' homes [3]. This lack of specific (and open) standards also makes it difficult and expensive for manufacturers to bring newer IPbased telecare systems to market [3]. Standardisation and thus interoperability issues have prevented consumer-driven mass production and investment in newer technologies [3]. Efforts however, have been made to harmonise standards for independent living applications and for medical information technology in general. This includes Personal Health Data (PHD) Standards (EN/ISO/IEEE 11073), which cover several aspects of defining medical data from device to server and defines a common framework for making personal health data available [18] and the ZigBee Health Care Application profile, which uses the 11073 PHD group of standards to promote interoperability. Additionally, the Continua Health Alliance, which is a non-profit alliance of healthcare and technology companies, selects technologies and standards and publishes guidelines to promote interoperability, which is guaranteed through its certification process [19].

Recent advances in sensor technologies and the social pressures for an increase in the utilisation of telecare constitute an incentive to develop new interoperable devices. The Technology Strategy Board's Assisted Living Innovation Platform funds the development of affordable and interoperable technology [20] and new Government policies encourage the convergence of telecare and telehealth [10]. 
Furthermore, the new FACS (Fair Access to Care Services) framework for allocating social care resources increases the number of tendered suppliers and thus competition and innovation [21].

\section{Future of Telecare: A Prediction}

The trends and issues discussed indicate a growing elderly population, putting a strain on care services, for which telecare offers a cost-effective solution (along with other health and social benefits). Problems with interoperability and infrastructure are being rapidly addressed and with strong demand, Government support, reducing technology cost and new innovative solutions, telecare will experience significant growth in the next decade (leading into the 2020s), both in its current form, and in newer forms, including IP-based technology and as part of a converged service offering.

\subsection{Investment and Infrastructure Development}

Following on from existing trends and given new Government policies which prioritise preventative technologies, health and social care strategies in the next decade will have a greater emphasis on, funding in and deployment of, telecare.

Large telecommunications providers are at present looking towards health technologies to revive a saturated broadband market and to drive growth in wireless services and will in the next 5-7 years, lead a trend of significant private investment in telecare, which will fuel the development of new technology. If telecare becomes a big customer for digital networks, there will be an upsurge in data-intensive telecare applications such as behaviour profiling and predictive modelling. An Analogy may be drawn with related developments in the digital delivery of multimedia content (IPTV), which has its roots in the significant investment by telecommunications providers in cable $\mathrm{TV}$ services in the 1990s and in high speed broadband in the 2000s.

Large multinational health technology companies are already investing heavily in the development of telecare technology and this will continue, leading to the emergence of consumeroriented mass-market products in telecare.

\subsection{Technology Development}

Assisted by greater penetration of high speed fixed and wireless broadband services, private infrastructure investment and eventually lower cost (as the technology starts to grow), IP-based technology will capture a sizeable proportion of the market share from analogue-based services by 2015-2018. Dual-mode technologies are already on offer from major suppliers, and these will remain popular as companies try to persuade a market wary of the reliability of an IP network.

\subsection{Standards and Interoperability}

Ongoing efforts to produce an IP standard for telecare will conclude by 2013-14, and it is envisaged that this will be adopted by most large telecare companies, owing to their membership of the TSA. Market leaders may however resist initial moves towards interoperability and may offer competing proprietary standards. It is expected however that given its membership size, the TSA-backed standard will gain eventual status as a UK (BSI) and eventually EU (EN) standard.

There are instances of open standards developments underway, such as those at the University of Portsmouth (FuTuRE) [22] and De Montfort University (iCare) [23] and it is predicted that these will offer a credible alternative to a proprietary or consortia-backed standard in that it will be free and open to community development. Smaller organisations may utilise an open-source model to reduce costs and improve time-to-market, leading to growth of a sizeable, but niche, open-source sector. This open-source development will additionally, fuel the development of 'fourth-generation' health technology, in the 2020s.

\subsection{Ubiquitous and Converged Services}

In the mid 2010s, mobile telecare will experience growth, fuelled by open standards such as paSOS and heading in to the 2020s, there will be a convergence of telemedicine, telecare and e-health technologies, resulting in greater integration of services and sharing of data. New informationbased services which integrate these technologies will allow health organisations to bridge the gap between clinical health, social care and general fitness, resulting in a truly universal health service which encompasses all elements of health and wellbeing management.

Research in Wireless Body Area Networks (WBAN) is ongoing and will by 2015, culminate in an international standard (IEEE 802.15.6). WBAN will pave the way for 'fourth-generation' technology which will combine wireless sensor networks with embedded or wearable health and general wellbeing devices. Research in this field is already being seen in publications and will gain stead in the marketplace as the technology moves from $R \& D$ to commercial deployment in the 2020s. Finally, the emergence of ubiquitous technology will lead to telecare devices thoroughly integrated into users' lives which can function both inside the home, as well as in remote locations.

\section{Conclusion}

Given an increasing elderly population with significant care needs and the associated cost savings of using telecare, there is an increasing impetus for Government to support the technology and put a greater focus on community care and for industry to develop next-generation equipment. However at the present rate of growth, telecare will not reach all of those who could benefit from using it, and there is a need for a more mainstreamed approach. Standards and interoperability 
issues are being addressed. The next decade will experience infrastructure development and marked private investment and it is predicted that new next-generation IP-technology will begin to be adopted. Open-standards and fourthgeneration WBAN-based technology will emerge in the 2020s, leading to the eventual convergence of remote health technologies, thoroughly integrated into users' lives.

\section{References}

1. Windmill, V. Caring for the Elderly. Harlow : Longman, 1996.

2. Office for Ntiaonal Statistics. Population Trends 139. 2010 .

3. Legal, Ethical and Socio-Economic Aspects of Community Telecare. Sethi, R., et al. London : IET, 2011. Assisted Living.

4. Department of Health. Building Telecare in England. 2005.

5. Yeandle, S. Telecare: a crucial opportunity to help save our health and social care system. s.l. : University of Leeds, 2009.

6. Care for older people in the United Kingdom: problems, provision and policy. Grundy, E. Costa Rica : LSE, 2010. Seminario: Experiencias internationalces y propuestas.

7. Williams, I. Telecare: A rapid review of the evidence 2005-2008. s.1. : NHS West Midlands and University of Birmingham, 2008.

8. Yeandle, S., Shipton, L. and Buckner, L. Local Challenges in Meeting Demand for Domiciliary Care: Synthesis Report. s.1. : Centre for Social Inclusion, 2006.

9. Office for National Statistics. Census 2001. 2001.

10. Age Concern. Telecare in Scotland. 2010.

11. The State of Telehealth and Telecare in the UK: Prospects for Integrated Care. Goodwin, N. 6, s.1. : Journal of Integrated Care, 2010, Vol. 18.

12. EU Communications Committee. Broadband access in the EU: situation at 1 July 2010. s.1. : EU Information Society and Media Directorate-General, 2010.

13. Van Dijk. Broadband Internet Access Cost. s.1. : EU Information Society and Media Directorate-General, 2010.

14. E-Health Insider. Telecare can grow to meet demands of 2020. [Online] 2501 2007. [Cited: 0712 2010.]

http://www.e-health-insider.com/news/item.cfm?ID=2441.

15. Jones, P. Triennial Review: Education (Lifelong Learning) - Internet Access and Use. s.1. : Equality and Human Rights Commission, 2010.

16. TSA. TSA and Continua Health Alliance Partner to Advance Healthcare. [Online] 1606 2010. [Cited: 1802 2011.] http://www.telecare.org.uk/information/45779/48672/48882/c ontinua_alliance_and_tsa/.

17. Social Services Improvement Agency. Investigation of the potential for the joint procurement of telecare equipment and maintenance. 2007.

18. ISO/IEEE. 11073-20601: Application Profile- Optimised Exchange Protocol. s.l. : IEEE, 2008.

19. Continua Health Alliance. The Next Generation of Personal Telehealth is Here. 2010.

20. TSB. Assisted Living. [Online] 2011. [Cited: 1802 2011.] http://www.innovateuk.org/ourstrategy/innovationplatforms/a ssistedliving.ashx.

21. Brand, D., Green, L. and Statham, D. Facts about FACS 2010: A guide to Fair Access to Care Services. s.1. : Social Care Institute for Excellence.

22. Interoperability and Standardisation in Community Telecare: A Review. Sethi, R., et al. London : IET, 2011. Assisted Living.

23. De Montford University. Homes Get Smart to Help Older People and Save Lives. [Online] 2209 2010. [Cited: 18 02 2011.]

http://dmu.ac.uk/news_events/news/archive/100922_iCARE.j sp. 